\title{
Encyclopedia
}

\section{Acoustic Waves at the Interface Between Different Types of Anisotropic-Strata}

Created by: Meishan Zhao

Version received: 4 January 2020

check for updates

Created by: Lin Fa and Meishan Zhao

\section{Introduction}

The acoustic waves at the interface between two different types of anisotropic media play an important role in acoustic applications, including geophysical applications [1, 2], seismic research [3, 4], petroleum logging [5-9], energy conversion device [5, 10], and medical imaging device [11].

A good model needs to be as simple as possible while remaining close to physical reality, such as the strata model inside the earth. The strata layers can be macro-anisotropic but transversely isotropic, where some are vertically symmetric and the others not [12]. The macroscopic anisotropy and related properties have been modeled based on the so-called elastic tensor, which is usually reserved for the hexagonal crystals $[1,2]$. Obviously, macroscopic anisotropy is significant for seismic waves with long wave-length regarding propagation, reflection, refraction, and polarization.

The refraction and reflection coefficients and their corresponding phase factors are the typical physical qualities that deserve special attention. Discussions have been focused mostly on and limited to the models of the transversely isotropic rock layers [13-18]. Due to mathematical complexity, there have been temptations and interests in approximate expressions [19-22]. However, the analytic solutions of the amplitudes of the reflected and refracted waves are desirable, yet difficult [23].

For angles of reflection and refraction, at the interface between similar types of strata, a fourth-order polynomial has been established for transversely isotropic media with a vertical axis of symmetry (VTI) [2425]. At the interface between unlike strata, an eighth-order polynomial has been established for transversely isotropic media with a tilting axis of symmetry (TTI).

While an interface between likewise-media has a relatively simple geometric structure [24-34], the interface between unlike-media can be much more complicated [35].

\section{The Interface Between VTI and TTI}

Sedimentary rocks are typically anisotropic media but treated as transversely isotropic. Mostly, they are polar anisotropic. The VTI and TTI media, as medium models, are the most common structures of the strata, as shown in Figure 1. At the interface between VTI and TTI media, the reflection and refraction coefficients are affected by many factors, including anisotropy, tilting-angle of rock-layer, and incidentangle.

Figure 2 is a schematic presentation of the interface between VTI and TTI media, showing the symmetric 
axes of the media and the geometric relationship between the VTI and TTI media with a TTI tilt-angle, $\varphi$.
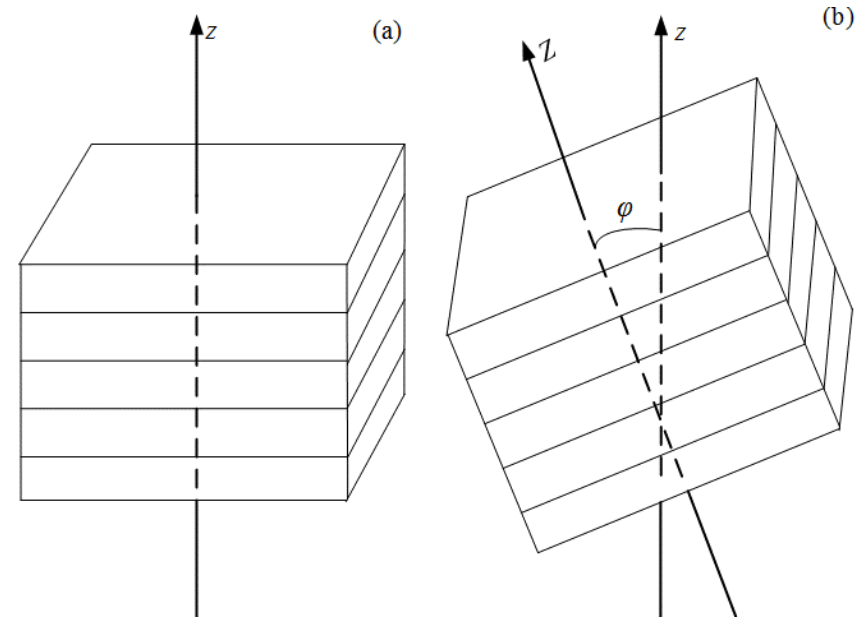

Figure 1. A schematic representation of the anisotropic rock media: (a) transversely isotropic media with a vertical axis of symmetry (VTI), (b) transversely isotropic media with a tilting axis of symmetry (TTI) [Ref. 35].

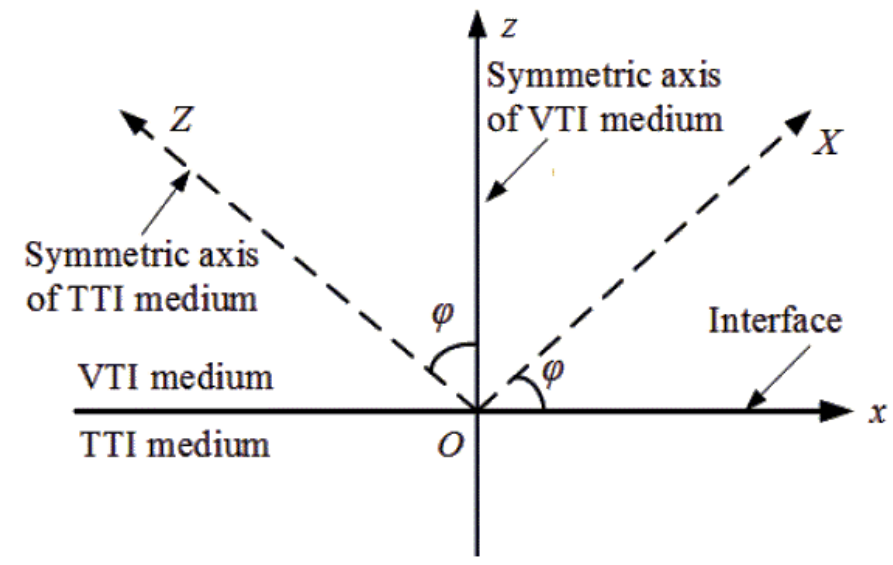

Figure 2. Schematic representation of VTI-TTI media interface, where Z-axis is the symmetric-axis of TTI medium; z-axis is the symmetric-axis of VTI medium, normal to the interface [Ref. 35].

An incident $P$-wave from the VTI medium striking on the interface would induce several new waves at the interface, e.g., reflected $P$-wave, reflected $S V$-wave, refracted $P$-wave, and refracted $S V$-wave, as shown in Figure 3.

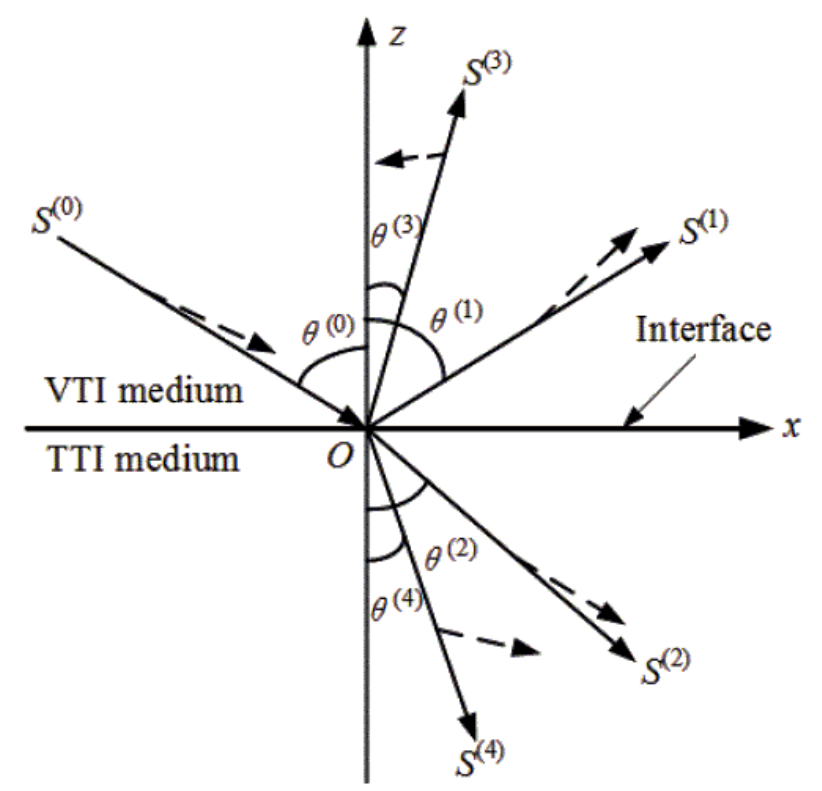


Figure 3. At the interface between VTI and TTI media, a $P$-wave from VTI medium leads to reflection and refraction, which produces the reflected $P$-wave, reflected $S V$-wave, refracted $P$-wave, and refracted $S V$ wave, corresponding to the index $(1,2,3,4)$ [Ref. 35].

The mechanical and physical properties of a general anisotropic medium can be described by an elastic stiffness tensor that may have up to 21 independent elements. The elastic stiffness tensors of the VTI and TTI media have only 5 independent elements [36, 37].

Now, consider an incident $P$-wave from VTI to TTI medium with an incident angle $(\theta)$. For the TTI medium, Bond transformation can be used to convert its tilt-angle structure to the same tensor form of the VTI structure [24-34]; Kelvin-Christoffel equation can be solved for the reflected and refracted coefficients; Snell's law can be applied to determine the angles of reflection and refraction and to guarantee energy conservation.

Inside the incident VTI medium, Snell's law yields a fourth-order polynomial for the angles of reflection of the reflected $P$-wave and $S V$-wave $[24,25]$,

$$
B_{1}^{(1,3)} \sin ^{4} \theta^{(1,3)}+B_{3}^{(1,3)} \sin ^{2} \theta^{(1,3)}+B_{5}^{(1,3)}=0 .
$$

Inside the refraction TTI medium, an eighth-order polynomial of the angles of refraction is obtained for the refracted $P$-wave and SV-wave [35],

$$
f_{1}^{(2,4)} \sin ^{8} \theta^{(2,4)}+f_{2}^{(2,4)} \sin ^{6} \theta^{(2,4)}+f_{3}^{(2,4)} \sin ^{4} \theta^{(2,4)}+f_{4}^{(2,4)} \sin ^{2} \theta^{(2,4)}+f_{5}^{(2,4)}=0
$$

In equations (1)-(2), $\theta$ is an incident angle; the coefficients $\left(B_{i}^{(1,3)}\right.$ and $\left.f_{i}^{(2,4)}\right)$ are functions of the incident angle, the anisotropy, and the mechanical parameters of the media.

\section{The Effects of TTI Tilting}

Consider the interface between VTI and TTI media, where the TTI medium has a tilting-angle, $\varphi$. The typical mechanical and anisotropy parameters for the VTI and TTI media have been reported in the literature [13$14,25,35,38-39]$. Typically, the tilting level of the TTI medium shows a significant influence on the critical incident angle, the reflection and refraction coefficients, the magnitude of power density flux, and the state of polarization.

The critical incidence angle $\left(\theta_{c}\right)$, corresponding to the refracted $P$-wave, has been affected significantly by the tilting level of the TTI medium and its anisotropy, as shown in Table 1. The tilting-angle and anisotropy of the TTI medium also influence the coefficients of the reflection and refraction. The calculated refraction coefficients and corresponding phase angles are shown in Figure 4.

Table 1: The critical incident angle versus the tilting-angle of TTI medium at the interface between VTI and TTI media.

$\begin{array}{llrr}\text { TTI tilting-angle, } \varphi & 0^{\circ} & 45^{\circ} & 60^{\circ} \\ \text { Critical incident-angle, } \theta_{C} & 41.3^{\circ} & 51.1^{\circ} & 55.3^{\circ}\end{array}$


It should be noted that the reflection and refraction coefficients are not smooth continuous at the angle of critical incidence.
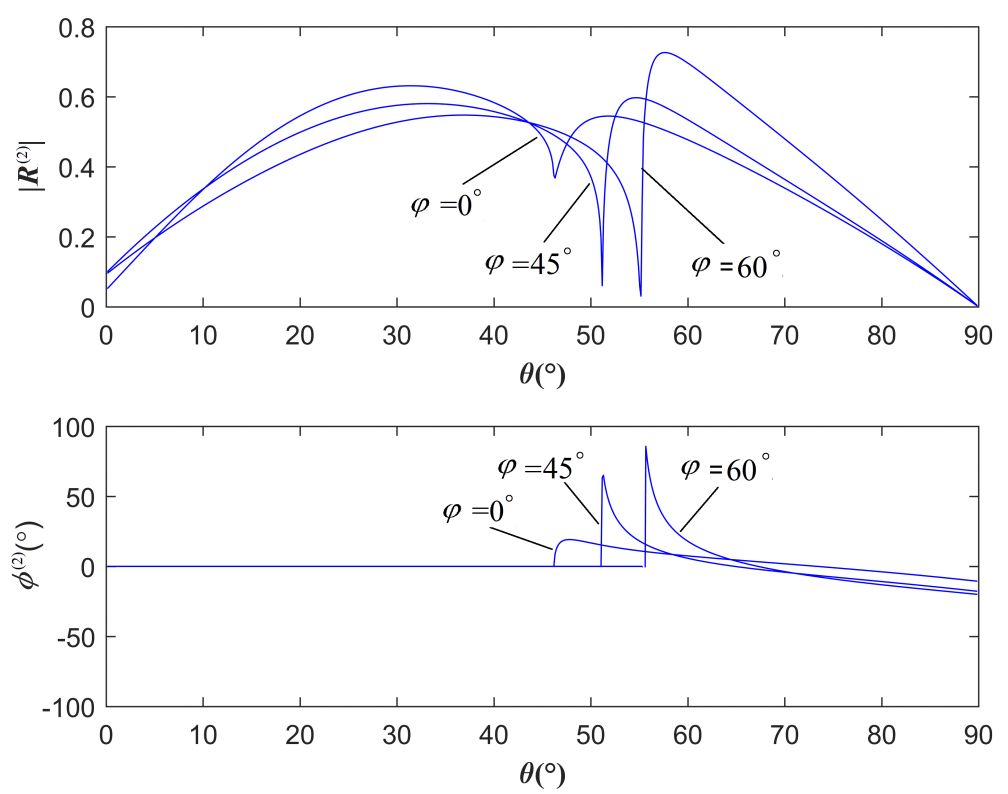

Figure 4. The amplitude and corresponding phase angle of the refracted wave, at the interface between VTI and TTI media, versus incident angle $(\theta)$ for three selected values of TTI tilting-angle $(\varphi)$ [Ref. 35].

The anisotropy influences the magnitude of power density flux but not the sum of the real parts of power density fluxes of the induced waves in the $z$-direction. The influence of the tilting-angle is even more significantly on the reflection and refraction coefficients, much more than anisotropy does. The real-parts of the energy density fluxes as a function of incident angle, for the induced waves in the $z$-direction, are calculated for several parameters and are presented in Figure 5. 

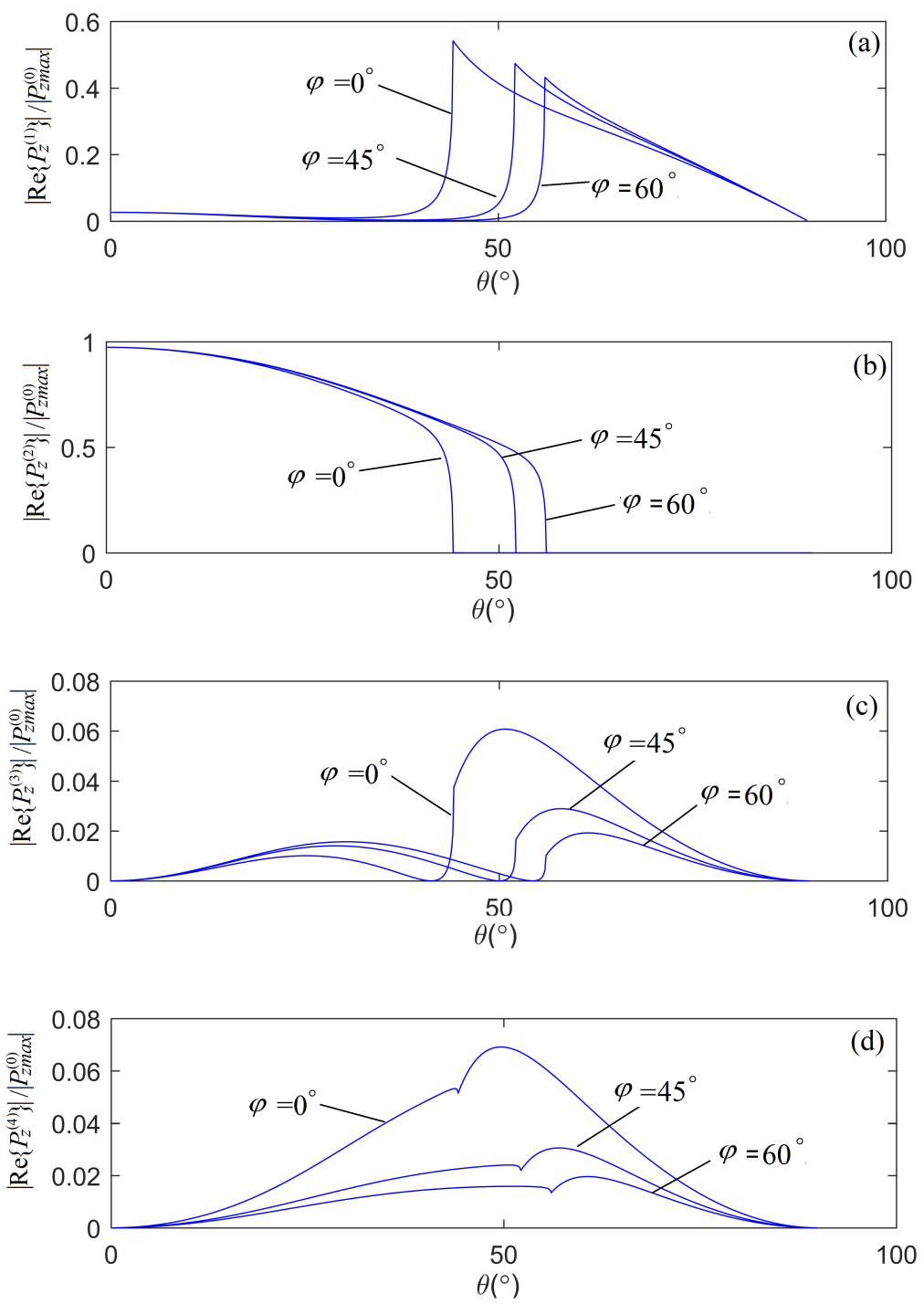

Figure 5. The energy density fluxes of the reflected and refracted waves perpendicular to the interface between VTI and TTI media versus incident angle $(\theta)$, for selected tilting-angles $(\varphi)$ of the TTI media. The blue, red, and black curves are for the cases of $T$ TI tilting-angle $\varphi=0^{\circ}, 45^{\circ}$, and $60^{\circ}$ [Ref. 35].

The polarization state of the refracted wave is also influenced by the tilting-angle and anisotropy of the refraction medium $[29,31,40]$.

Prior to the critical incident angle, the polarization coefficients of the waves are all mathematically real numbers, including the incident $P$-wave, reflected $P$-wave, reflected $S V$-waves, refracted $P$-wave, and refracted SV-wave.

In the post-critical angle region, except for the refracted $P$-wave, the polarization coefficients are complex numbers for all waves. The polarization coefficients of the refracted $P$-wave have two possible solutions. Each solution has two independent components $\left(u_{x}^{(2)}\right.$ and $\left.u_{z}^{(2)}\right)$, where one component is purely real; the other is purely imaginary. If the $x$-component is purely real, the $z$-component must be purely imaginary; vice versa. The choice of the solution determines the direction of polarization.

\section{Final Comments}

Based on our current knowledge from the studies of the VTI-VTI anisotropic media interface, the VTI-TTI interface can be explained, through Bond transformation, by borrowing the techniques used in the VTI-VTI 
interface. Additional work will lead to improved understanding of the interface between different types of anisotropic media, such as the interface between TVI and TTI anisotropic media. In practical applications for energy conversion, on the transient response of the acoustic-electric transducers, a similar issue may appear for acoustic scattering from spheres and cylinders [41-43].

The next steps may include the determinations of anomalous refraction if it ever exists, the effect of the competition between anisotropy and tilting-angle of the media, and the effect of anisotropy and the tiltingangle on polarization conversion. With respect to reflection and refraction, it is extremely desirable to achieve a better understanding of the relative importance of the anisotropy and TTI angle tilting, i.e., the competition between the anisotropy and angle-tilting of the media.

\section{References}

1. L. Thomsen, “Weak anisotropy," Geophysics 51, 1954-1966 (1986).

2. I. Tsvankin, "P-wave signatures and notation for transversely isotropic media: an overview," Geophys. 61, 467-483 (1996).

3. J. P. Castagna and M. M. Backus, "Offset-Dependent Reflectivity-Theory and Practice of AVO Analysis," Society of Exploration Geophysicists,

4. L. Fa, L. Xue, Y. X. Fa, Y. L. Han, Y. D. Zhang, and H. S. Cheng. F. Ding, G. H. Li, S. J. Tang, C. L. Bai, B. J. Xi, X. L. Zhang, and M. S. Zhao, “Acoustic Goos-Hänchen effect," Sci. China. Phys. Mech. 66, 104311 (2017).

5. R. J. Przybyla, H. Y. Tang, A. Guedes, S. E. Shelton, D. A. Horsley, and B. E. Boser, “3D ultrasonic rangefinder on a chip," IEEE Journal of Solid-State Circuits 50, 320-334 (2015).

6. M. A. Breazeale, L. Adlerand, and G. W. Scott, "Interaction of ultrasonic waves incident at the Rayleigh angle onto a liquid-solid interface," J. Appl. Phys. 48, 530-537 (1977).

7. A. Atlar, C. F. Quate, and H. K. Wickramasinghe, "Phase imaging in reflection with the acoustic microscope," Phys. Lett. 31, 791-793 (1977).

8. R. Briers, O. Leroy, and G. Shkerdin, "Bounded beam interaction with thin inclusions. Characterization by phase differences at Rayleigh angle incidence," Acoust. Soc. Am. 108, 1622-1630 (2000).

9. G. L. Zhang, C. T. Hao, and C. Yao, "Analytical study of the reflection and transmission coefficient of the submarine interface," Acta Geophys. 66, 449-460 (2018).

10. M. A. Mustapa, O.B.Yaakob, Y. M. Ahmed, C. Rheem, K. K. Koh, and F. A. Adnan, Renew, "Wave energy device and breakwater integration: A review," Renew. Sust. Energ. Rev. 77, 43-58 (2017).

11. A. S. Goh, J. C. Kohn, D B. Rootman, J. L. Lin, and R. A. Goldberg, Surg J., 2014, 34: 510-515.

*****************

12. G. L. Backus, “Long-wave elastic anisotropy produced by horizontal layering,” J. Geophys. Res. 67, 44274440 (1962).

13. P. F. Daley, and F. Hron, "Reflection and transmission coefficients for transversely isotropic media," Bullet. S Society. Am. 67, 661-675 (1977).

14. P. F. Daley, and F. Hron, "Reflection and transmission coefficients for seismic waves in ellipsoidally anistropic media," Geophys. 44, 27-38 (1979).

15. A. Rüger, "P -wave reflection coefficients for transversely isotropic models with vertical and horizontal axis of symmetry," Geophys. 62, 713-722 (1997).

16. I. Tsvankin, Seismic Signature and Analysis of Reflection Data in Anisotropic Media, Elsevier, Amsterdam: Society of Exploration Geophysicists, 2005.

17. J. M. Carcione, Wave Fields in Real Media: Wave Propagation in Anisotropic, Anelastic And Porous Media, Elsevier Science, 2001.

18. L. Klimeš, "Weak-contrast reflection-transmission coefficients in a generally anisotropic background," Geophys. 68, 2063-2071 (2003).

19. P. G. Richards and C. W. Frasier, "Scattering of elastic waves from depth-dependent inhomogeneities," 


\section{Encyclopedia}

41, 441-458 (1976).

20. K. Aki and P. G. Richards, "Theory and Methods," in Quantitative Seismology, H. Freeman, New York, 1980.

21. R. T. Shuey, "A simplification of the Zoeppritz equations," 50, 609-614 (1985).

22. W. J. Ostrander, "Plane-wave reflection coefficients for gas sands at nonnormal angles of incidence," 49, 1637-1648 (1984).

23. K. Zoeppritz, "On the reflection and penetration of seismic waves through unstable layers," Gottinger $\mathbf{1}$, 66-84 (1919).

24. L. Fa, R. Brown, J. Castagna, “Anomalous postcritical refraction behavior for certain transversely isotropic media," J. Acoust. Soc. Am. 120, 3479-3492 (2006).

25. L. Fa, J. Castagna, H. Dong, "An accurately fast algorithm of calculating reflection/transmission coefficients." Sci. China-Phys. Mech. Astron. 51, 823-846 (2008).

26. Y. Zhao, N. Zhao, L. Fa and M. Zhao, "Seismic signal and data analysis of rock media with vertical anisotropy," J. Modern Phys. 4, 11-18 (2013), doi:10.4236/jmp.2013.41003.

27. L. Fa and M. Zhao, "Recent progress in acoustical theory and applications," in Understanding Plane Waves, Nova Science Publishers, Chapter 1, pp. 1-114, 2019.

28. L. Fa and M. Zhao, "Recent development of an acoustic measurement system," in Understanding Plane Waves, Nova Science Publishers, Chapter 2, pp. 115-147, 2019.

29. L. Fa, J. Zhao, Y. Han and M. Zhao, "Polarization state of an inhomogeneously refracted compressionalwave induced at interface between two anisotropic-rocks," J. Acous. Soci. Amer. 141, 1 (2017), doi:10.1121/1.4973410.

30. L. Fa, J. Zhao, Y. Han, G. Li, P. Ding and M. Zhao, "The influence of rock anisotropy on the ellipticalpolarization state of inhomogeneously refracted P-wave," Science China Physics, Mechanics \& Astronomy 59, 644301 (2016), doi:10.1007/S11433-015-00441-1.

31. L. Fa, Y. Fa, Y. Zhang, P. Ding, J. Gong, G. Li, L. Li, S. Tang and M. Zhao, “Anomalous incident-angle and elliptical-polarization rotation of an elastically refracted P-wave," Scientific Reports 5, 12700 (2015), doi:10.1038/srep12700.

32. L. Fa, J. P. Castagna, Z. Zeng, R. L. Brown and M. Zhao, “Effects of anisotropy on Time-Depth Relation in Transversely Isotropic Medium with a Vertical Axis of Symmetry," Chin. Sci. Bulle. 55, 2241 (2010). doi: 10.1007/s11434-010-3186-4.

33. M. Zhao and L. Fa, "The Goos-Hänchen Effect: An Intriguing Phenomenon from Optics to Acoustics," Encyclopedia, 2019, v1, Available online: https://encyclopedia.pub/386.

34. L. Fa and M. Zhao, "Network Modeling of Piezoelectric Transducers for Energy Conversion," Encyclopedia, 2019, v1, Available online: https://encyclopedia.pub/367.

35. L. Fa, J. Tang, Q. Zhang, M. Zhang, Y. Zhang, M. Liang, and M. Zhao, "Reflection and refraction of acoustic wave at VTI-TTI media interface," Frontiers of Physics 15, 22601 (2020).

36. P. Burnley, "Tensors: Stress, Strain and Elasticity," Teach the Earth the portal for Earth Education, 2012. http://serc.carleton.edu/NAGTWorkshops/mineralogy/mineral_physics/tensors.html

37. D. Leng, L. Sun, J. Sun, and Y. Lin “Derivation of stiffness matrix in constitutive modeling of magnetorheological elastomer," J. of Phys.: Conference Series 412, 012028 (2013).

38. J. Cai, Y. He, Z. Li, B. Wang, and M. Guo, "TTI/VTI anisotropy parameters estimation by focusing analysis, Part I: theory," https://doi.org/10.1190/1.3255480.

39. J. Cai, Y. He, Z. Li, B. Wang, and M. Guo, "TTI/VTI anisotropy parameters estimation by focusing analysis, Part II: application," https://doi.org/10.1190/1.3255504.

40. L. Fa, M. Zhao, J. P. Castagna, Y. C. Liu, L. Wang, Y. Q. Wang and J. G. Sun, “On polarization of plane wave propagating inside elastic hexagonal system solids," Science China: Physics, Mechanics \& Astronomy 57, 1-12 (2014), doi: 10.1007/s11433-013-5363-3.

41. L. Fa, X. Zhou, Y. X. Fa, Y. D. Zhang, J. P. Mou, M. Liang, M. M. Wang, Q. Zhang, P. F. Ding, W. T. Feng, H. Yang, M. S. Zhao, "An innovative model for the transient response of a spherical thin-shell transducer and an experimental confirmation," Sci. China Phys. Mech. Astron. 61, 114321 (2018). https://doi.org/10.1007/s11433-018-9236-X.

42. L. Fa, N. Tu, H. Qu, Y. G. Wu, K. Sun, Y. D. Zhang, M. Liang, X. G. Fang, and M. Zhao, "Physical 


\section{Encyclopedia}

characteristics of and transient response from thin-cylindrical piezoelectric transducers used in petroleum logging tool," Micromachines 10, 804 (2019). doi:10.3390/mi10120804.

43. L. Fa, J. P. Mou, Y. X. Fa, X. Zhou, Y. D. Zhang, M. Liang, P. Ding, S. Tang, H. Yang, Q. Zhang, M. M. Wang, G. H. Li, and M. Zhao, “On transient response of piezoelectric transducers," Front. Phys. 6, 123 (2018). doi.org/10.3389/fphy.2018.00123.

\section{Keywords}

VTI medium; TII medium; rock anisotropic media; transversely isotropic media; VTI-TTI interface; TTI tilting-angle; incident critical-angle; reflection; refraction 